ISSN: $1130-3743$

\title{
TEORÍA DE LA EDUCACIÓN Y CIENCIAS DE LA EDUCACIÓN: CARÁCTER Y UBICACIÓN
}

\section{THEORY OF EDUCATION AND SCIENCES OF EDUCATION: CHARACTER AND POSITION}

\author{
ANTONIO J. COLOM CAÑELLAS, Mª DEL PINO RODRÍGUEZ CRUZ \\ Facultad de Educación. Universidad de las Islas Baleares. Campus Universitario. 07071 Palma \\ de Mallorca. Centro Superior de Formación del Profesorado. Universidad de las Palmas de G.C. \\ C/. Juana del Arco, s/n. 35004 Las Palmas de G. Canarias.
}

\section{RESUMEN}

En este artículo se analiza la estructura interna de las ciencias de la educación en su sentido sincrónico y diacrónico y también sus relaciones externas con las ciencias humanas, siguiendo para ello el modelo que Piaget plantea de las mismas. De ello se desprende una concepción centrada en la subsidiariedad y en la complementariedad, por lo que entre las ciencias de la educación y las ciencias humanas se integra un modelo abierto y circular de mutua influencia.

Al mismo tiempo se analiza la posición que ocupa la Teoría de la Educación, concluyendo que su carácter está centrado en la articulación entre la teoría y la práctica, por lo que es definida como una teoría tecnológica.

\section{SUMMARY}

In this article it analyses the internal structure of education sciences in synchronic and dyacronic sense, as well as the externals relations with humans sciences following a piagetian model. From there it transpires a conception centered in the subsidiaryty and in the complementaryty, for that education sciences and human sciences shelter an openning and circulate model of mutual influence.

At the same time it analyses the position that occupies the Theory of Education, concluding that its character is centered in the articulation between the theory and the practice, for that it defineted as a technologycal theory. 


\section{Algunas CUESTIONES INICIALES}

En un trabajo previo ${ }^{1}$ se planteaba el origen o génesis de la Teoría de la Educación mencionando dos grandes desmembraciones que se habían producido en el cuerpo de conocimientos de la Pedagogía General, de marcado carácter herbartiano, y que sin duda fue el constructo en el que se amalgamó el conocimiento acerca de la educación durante el siglo XIX. La primera de estas desmembraciones a las que hacíamos referencia fue de índole metodológica, al surgir, fundamentalmente de manos de Lay, la posibilidad de aplicar el método experimental a la cuestión educativa. De esta manera, el monolitismo metodológico centrado en la racionalidad, propia de la filosofía, y que daba origen a la Pedagogía Racional, se dualizaba con la aparición de la Pedagogía Experimental.

De todas formas, más determinante al menos para nuestros fines, fue la segunda desmembración que tuvo lugar, fundamentalmente, tras la segunda guerra mundial, si bien ya con anterioridad, en la década de los años treinta, había hecho su aparición de manera más que insinuante. En esta ocasión, lo que se desgajaba era el propio cuerpo de la Pedagogía General, debido en parte al afan especializador y también al interés que otras ciencias o disciplinas mostraban por las cuestiones educativas, surgiendo en consecuencia, múltiples enfoques, que pronto exigieron un espacio propio cuando no una fundamentación epistemológica diferenciada.

Fueron, como se sabe, las diferentes ciencias humanas las que en su fulgurante desarrollo coincidieron en considerar la educación como un espacio propio de sus respectivos estudios, en el fondo, muy en la línea de Biología, que ya a finales del XIX había hecho lo propio hasta tal punto que consideraba a la Pedagogía como un capítulo más de la Biología, el que se ocupaba de la crianza y adecuado desarrollo del animal humano, para expresarlo con palabras caras a Spencer, uno de los máximos exponentes de tal postura. Así pues, y con tal precedente, no debe extrañarnos que en un primer momento la Filosofía se resistiese a abandonar una parcela que desde Aristóteles y Platón había pertenecido a su ámbito, y que la Psicología, vistas sus alianzas a través de la Paidología con las cuestiones educativas, se afanase por considerar asimismo gran parte de la Pedagogía como objeto de su estudio.

A partir de aquí, la Sociología, - recuérdese a Durkheim y su discurso con motivo de la inauguración del curso 1901-1902 en la Sorbona ${ }^{2}$ - y también la Antropología 3 , se decantarán como materias con capacidad de estudio de las cuestiones pedagógicas. La puerta evidentemente estaba ya abierta, y sólo será el tiem-

1. Nos referimos a Colom, A. J.: "El saber de la Teoría de la Educación", pp. 11 a 19 de Teoría de la Educación, vol. IV, Salamanca, 1992.

2. Se trata del famoso texto de Durkнeim "Pedagogía y Sociología" que luego se publicaría en la Revue de Metaphysique et Morale, de París, en su número de enero de 1902 y que es considerado como el fundamento conceptual de la Sociología de la Educación. En castellano puede encontrarse por ejemplo en DuRKheim, E.: Educación y Sociologia, Edit. Península, Barcelona, 1975, pp. 95 a 116.

3. Recuérdese como ejemplo el libro de MonTESSORI, M. publicado en Roma en 1913, y titulado expresivamente Antropología pedagógica. 
po y el ejemplo que hará que otros desarrollos científicos, de principio extraños a la Pedagogía, se aproximen a ella a fin de exigir para sí parcelas de su estudio y aplicación. La economía, el derecho, la psiquiatría, la ciencia de la organización... etc. sólo forman parte de algunos de los más conocidos casos que confirman el fenómeno que estamos insinuando.

La Pedagogía quedaba reducida a muy poca cosa, máxime cuando en su seno también cundió la subdivisión y la independencia de los escasos núcleos temáticos que podían considerarse como propios. La Historia de la Pedagogía lo tuvo muy fácil por cuanto su metodología le daba una distinción específica y unos fundamentos epistemológicos absolutamente diferenciados, por lo que de aquella antigua tradición, incluso preherbartiana, de iniciar los libros referidos a la educación con referencias a las teorías históricas sobre la Pedagogía surgió otra nueva disciplina que desde entonces y con absoluta independencia se planteó el estudio histórico de todas las cuestiones que afectan a la educación. Junto con ella, la Didáctica, y a través de los autores seguidores de Herbart - Rein, Ziller, y O. Willman- fue también decantándose y adquiriendo independencia y fundamentación propia, al centrarse en concreto sobre las cuestiones pertinentes a la enseñanza, al aprendizaje y los métodos congruentes con ambos fenómenos.

Sólo hemos querido referenciar algunas notas y aspectos que creemos son suficientes para evidenciar el fenómeno que ha conllevado a la sustitución progresiva de la Pedagogía General por las Ciencias de la Educación en la segunda mitad del presente siglo, y al mismo tiempo, para insistir, en la necesidad de integrar este desarrollo plural dentro del contexto epistemológico de la teoría educativa, y vislumbrar también qué lugar ocupa, entre ellas, la Teoría de la Educación, tareas éstas a la que a continuación vamos a dedicar nuestra atención.

\section{Caracterización de las Ciencias de la Educación}

Hablar de las Ciencias de la Educación no supone referirse a comportamientos estancos, e independientes; tampoco se trata de establecer conexiones fidedignas y formalizadas, aunque la verdad, al incidir todas ellas en un aspecto común - la educación y lo educativo - muestran realmente puntos y núcleos de conexión e incidencia. No es de extrañar que se haya afirmado y patentizado, incluso entre nosotros, el carácter abierto ${ }^{4}$ del conocimiento pedagógico, o con una pretensión idéntica, su concepción como sistema circular que "atienda a la interconexión necesaria entre los diferentes campos, objetos y métodos susceptibles de proporcionar esquemas más apropiados a la explicación de una realidad, no parcelada, sino interactuante" (Pérez Gómez, 1978, 64).

Gracias al carácter abierto de las ciencias de la educación puede establecerse este sistema circular que conforma un auténtico sistema de comunicación entre

4. Tal como explícitamente puede leerse en Colom, A. J.: Teoría y metateoría de la educación, Edit. Trillas, México, 1982. 
todos los aportes que tales ciencias nos ofertan, permitiendo, además, introducir las innovaciones externas que contribuyen al desarrollo del conocimiento. Es, además, gracias a esta abertura y su consecuente realimentación en un sistema circular de todos los conocimientos acerca de la educación, lo que nos permite al mismo tiempo hablar y postular la existencia de la Teoría de la Educación, de carácter eminentemente integrador.

Ahora bien, si nuestro objetivo es clarificar el sentido epistemológico de las Ciencias de la Educación, no hay duda que debemos buscar y encontrar un nivel de pensamiento que sea capaz de abarcar y de dar razón común de la diversidad y diferencia que el sentido plural de las propias Ciencias de la Educación nos manifiestan. O sea, hay que profundizar más a fin de encontrar una base sólida que dé y aporte un sustrato común a la diferenciación implícita en tal denominación. Para ello, necesariamente, tendremos que definir qué tipo de ciencias conforman la constelación plural de saberes integrados en las Ciencias de la Educación, y evidentemente, no hay duda que en este sentido podemos distinguir diversas dimensiones que coinciden a la hora de entender y definir el fenómeno educativo - y por tanto su conocimiento- por lo que contaminan y caracterizan a las ciencias que se aplican al ámbito educativo-pedagógico. Podemos hablar pues de:

- una dimensión formal, de carácter deductivo y lineal, dificilmente constatable en la realidad ya que emerge desde la consideración ideológica o metodológica de la propia educación,

- una dimensión fáctica que se centraría en estudiar las manifestaciones educativas que se dan en la realidad, por lo que nos es posible establecer entre ellas relaciones de causa y efecto,

-- una dimensión filosófica, a través del pensamiento reflexivo, que nos mostraría el ideal o el que debiera ser la educación como camino para comprender y conocer qué es verdaderamente la educación,

Esta división que Pérez Gómez recogiera en su día bien puede servirnos para advertir que en las Ciencias de la Educación se entremezclan los saberes formales, los fácticos y los filosóficos, por lo que queda aun más marcada si cabe su acentuada y plural complejidad; claro que también y al mismo tiempo nos da la oportunidad de sistematizar los puntos hasta aquí considerados como características propias de las Ciencias de la Educación y que a nuestro entender serían los siguientes:

- Paradigma multidimensional centrado en un objeto plural de conocimiento - la educación - que a su vez es tratado desde una pluralidad de disciplinas externas.

- Sistema abierto y circular de información.

- Aportes de conocimientos formales, fácticos y filosóficos que basculan sobre el concepto de educación.

Así pues, afrontar el tratamiento epistemológico de las Ciencias de la Educación supone forzosamente incidir en dos planteamientos; el primero, debe atender a la 
dimensión interna de estas ciencias en su doble aspecto sincrónico y diacrónico. El segundo, deberá por su parte integrar las Ciencias de la Educación en el cuadro general de las ciencias humanas (y de otras colaterales, como la Biología), todo ello relacionando y ubicando al mismo tiempo el espacio propio de la Teoría de la Educación. Pues bien, tal dimensionalidad nos permite hablar respectivamente para el primer y segundo caso de:

- Endoestructura de las Ciencias de la Educación.

- Exoestructura de las Ciencias de la Educación.

Intentaremos ahora y en primer lugar, clasificar la endoestructura de las Ciencias de la Educación bien entendido que tal fin implica una doble perspectiva, a saber, la sincrónica que nos obliga a un análisis estático de la cuestión, y la diacrónica que implica plasmar su sentido evolutivo, procesual, y en definitiva, dinámico.

\section{a) Perspectiva estática o sincrónica de la endoestructura de las Ciencias de la Educación}

Desde tal planteamiento consideramos que cabe distinguir dos grandes núcleos de Ciencias de la Educación en función del papel que jueguen sus contenidos; así, distinguiríamos claramente entre disciplinas fundamentantes y específicas o concretas, de acuerdo con la interpretación que realizan del hecho educativo.

Los contenidos fundamentales vendrían dados por todas las disciplinas educativas que aportan claridad en los factores que intervienen en la aparición y génesis de las explicaciones acerca de la educación y de los procesos educativos, tal como puedan ser, conceptos, teorías y modelos. Abarcarían aquellas Ciencias de la Educación que se convierten en puntales y basamento del conocimiento científico de la educación, del conocimiento ideológico, y del conocimiento histórico. En definitiva, son ciencias fundamentales porque nos dicen lo que es la educación (perspectiva científico-realista), lo que podría o debería ser la educación (perspectiva especulativo-filosófica) o lo que fue la educación (perspectiva historicista). También podríamos añadir, el conocimiento prospectivo, que nos aportaría información sobre que será la educación en el futuro.

En cambio, entendemos por contenidos específicos aquellos que se centran en la dimensión más práctica del hacer educativo ya que tienen como finalidad producir efectos. Como el lector podrá comprobar resumimos la funcionalidad de las materias fundamentales en el saber acerca de la educación, mientras que la nota determinante de las materias específicas será el hacer. Pues bien la síntesis de ambos planteamientos nos posibilitará la ubicación de la Teoría de la educación dentro del amplio entramado de las Ciencias de la Educación. Para nosotros, efectivamente, la Teoría de la Educación se plantea como una teoría - saber- que encuentra su razón de ser en su aplicabilidad —en el hacer-. Con ello mantenemos el carácter unitario y sintetizador que consideramos es el rasgo fundamental de la Teoría de la Educación entendida como una de las Ciencias de la Educación. Hemos querido plasmar nuestra opción en el diagrama que introducimos a continuación: 
CuAdro 1. Perspectiva estática o sincrónica de la endoestructura de las Ciencias de la Educación

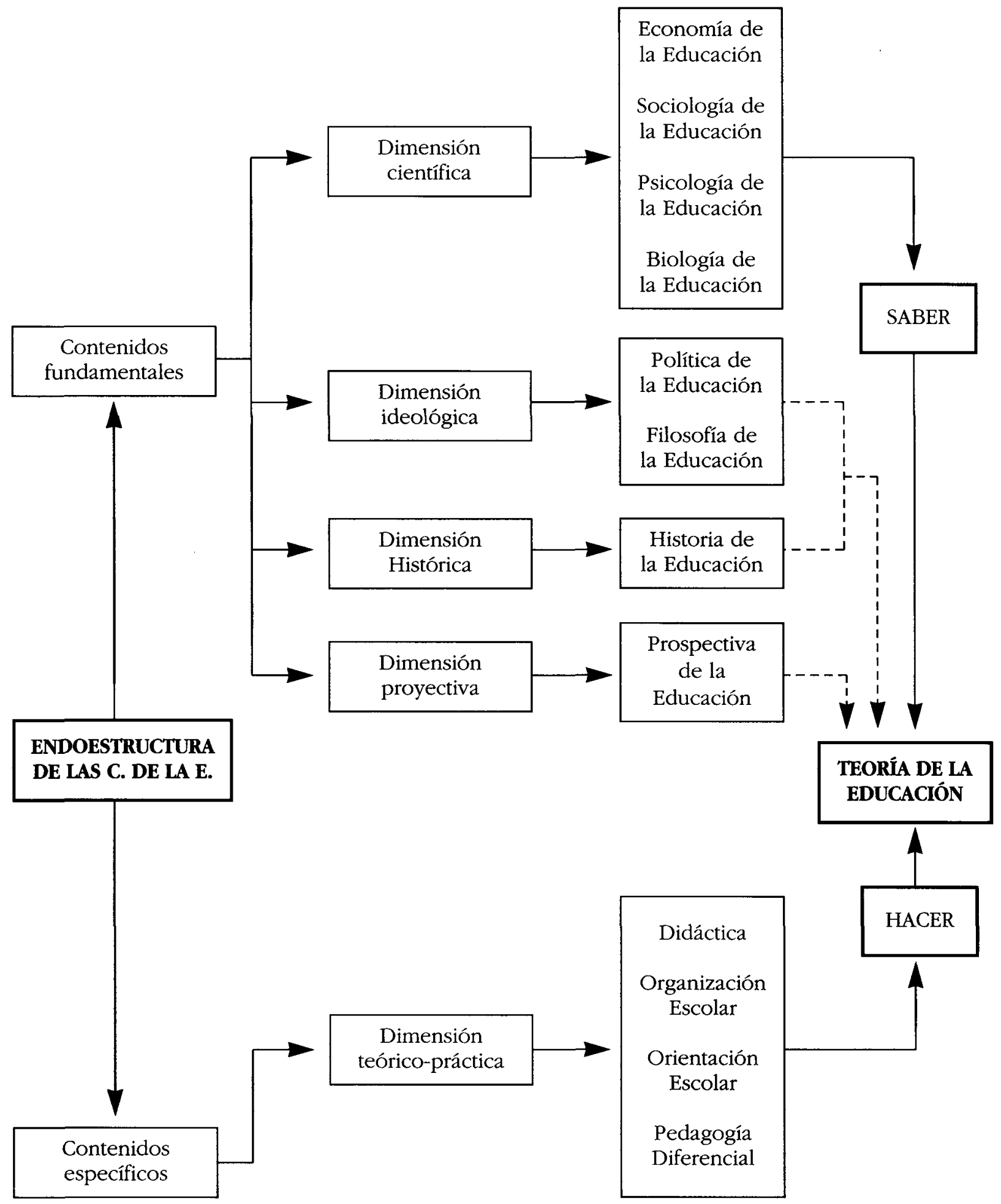

Relaciones fundamentales.

Relaciones auxiliares u orientativas.

(C) Ediciones Universidad de Salamanca

Teor. educ. 8, 1996, pp. 43-54 
b) Perspectiva dinámica o diacrónica de la endoestructura de las Ciencias de la Educación

En la consideración diacrónica existen unas relaciones bidireccionales de tal modo que la teoría y la práctica educativa generan entre sí procesos de interaciones mutuas de tal manera que los presupuestos teóricos pueden condicionar el posicionamiento práctico así como el heurístico o investigador, si bien, al mismo tiempo, los resultados evaluados de tal práctica pueden generar alteraciones en las teorías acerca de la educación y por tanto en alguna o diversas Ciencias de la Educación. Hemos intentado, manteniendo siempre la ubicación de la Teoría de la Educación en el complejo entramado de las Ciencias de la Educación, manifestar gráficamente nuestra posición a partir del diagrama que también introducimos en el Cuadro 2. De él destacaría el papel destacado que juega para la acción educativa la Teoría de la Educación, que se nos presenta como un saber articulante y conexionador de la teoría y la práctica en la educación. Es importante pues manifestar que este mismo papel tendría que jugar en sus desarrollos teóricos y bibliográficos, lo que sabemos no ocurre aun entre nosotros de forma generalizada.

\section{c) Exoestructura de las Ciencias de la Educación}

Tal como habíamos manifestado, nos referiremos ahora, para así completar nuestro propósito, al carácter y ubicación de las Ciencias de la Educación en relación a las ciencias humanas, o sea, pretendemos plantear su perspectiva externa o exoestructura. Para ello, necesariamente requerimos ajustar las Ciencias de la Educación a alguna categorización de las ciencias humanas, lo que haremos basándonos en la reconocida clasificación que de las mismas realiza J. Piaget en sus trabajos sobre epistemología.

La elección de tal autor, por supuesto subjetiva al igual que hubiese ocurrido con la elección de otras taxonomías, obedece en todo caso a su predicamento dentro del propio contexto de las Ciencias de la Educación al desarrollar gran parte de su obra en unos terrenos muy próximos a los intereses pedagógicos. En efecto, sus aportaciones a la psicología evolutiva, fundamentalmente en las etapas infantiles y adolescente, hace que sus estudios se ubiquen en terrenos muy próximos a los psicopedagógicos.

Por otra parte, sus críticas epistemológicas vertidas a la Pedagogía 5 , hace que sintamos la necesidad de superar tales posicionamientos; en este sentido, cabe destacar que Piaget se refiere en la década de los sesenta a la Pedagogía, en su sentido decimonónico, sin atender al fenómeno que concurría paralelamente a su escrito, de aparición y consolidación de la amplia constelación de saberes englobados bajo la denominación Ciencias de la Educación. Si hubiere atendido a este último aspecto es indudable que el saber pedagógico no hubiese quedado en absoluto tan mal parado a la hora de razonar sobre su consistencia conceptual.

5. En este sentido sería preciso consultar su libro Psicología y Pedagogía, Edit. Ariel, colección Ariel quincenal, no 26, Barcelona, 1969.

(C) Ediciones Universidad de Salamanca

Teor. educ. 8,1996 , pp. $43-54$ 
CuAdRo 2. Perspectiva dinámica o diacrónica de la endoestructura de las Ciencias de la Educación.

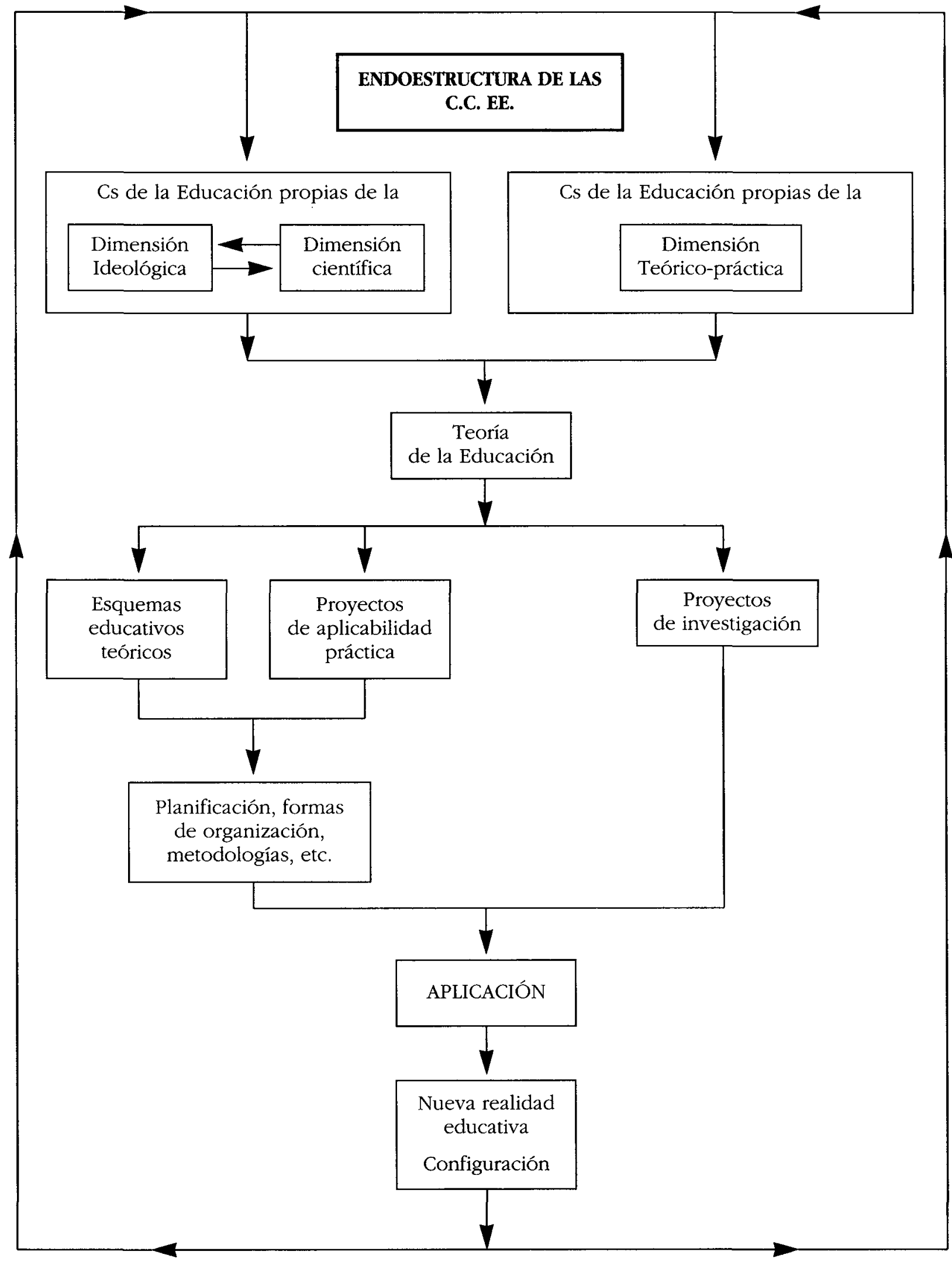

Ediciones Universidad de Salamanca

Teor. educ. 8,1996 , pp. 43-54 
Estas pues han sido, en todo caso, las dos razones que defienden nuestro subjetivismo a la hora de plantear la exoestructuración de las Ciencias de la Educación a la luz del pensamiento de Piaget, a saber, la proximidad de su obra a las cuestiones pedagógicas, y su crítica, no del todo matizada, que en un momento dado realizara a la Pedagogía. Para llevar a cabo nuestro objetivo plantearemos en un mismo esquema y al mismo tiempo, el tipo de ciencias según Piaget y sus desarrollos en el ámbito de las Ciencias de la Educación ${ }^{6}$.

No debemos olvidar que la clasificación que Piaget nos aporta de las diversas ciencias humanas hace que éstas sean entendidas, consideradas y diferenciadas de acuerdo a su carácter nomotético, histórico, normativo y filosófico. Pues bien, una aplicación de tal taxonomía a las Ciencias de la Educación nos daría las siguientes perspectivas:

- Dimensión nomotética de las Ciencias de la Educación: Son ciencias que se preocupan u ocupan del establecimiento de las leyes que clarifiquen los fenómenos. En nuestro caso es indudable que los fenómenos educativos comportan una dimensión descriptivo-explicativa, al intentar estudiar todos los casos que acaecen así como todos los factores que concurren hasta la enunciación de la ley. Pertenecerán a este grupo todas aquellas disciplinas que buscan una consolidación científica del saber pedagógico-educativo.

- Dimensión histórica de las Ciencias de la Educación: Indudablemente el condicionamiento del pasado se da y se denota en cualquier manifestación disciplinar de las ciencias humanas, y por ende lo mismo sucederá en el caso de las Ciencias de la Educación, ya que solo una visión diacrónica de los fenómenos educativos nos puede mostrar el sentido de tales condicionamientos e incidencias tanto en el terreno de lo teórico como en el de la práctica educativa.

- Dimensión normativa de las Ciencias de la Educación: Se puede afirmar, sin gran peligro por nuestra parte, que lo normativo es consustancial a lo pedagógico y por lo tanto también y en parte a las Ciencias de la Educación. Ahora bien, la normatividad que se busca en nuestro contexto no es atemporal ni acrítica ya que debemos solicitar como característica ineludible de tal normatividad el control y encauzamiento de los factores que en cada momento pueden condicionar los acontecimientos educativos así como la orientación de las actividades educativas en función del "deber ser" de la educación.

- Dimensión filosófica de las Ciencias de la Educación, o materias que partiendo de la concepción del hombre y del mundo plantean exactamente los condicionamientos y características de este "deber ser" educativo que debe servir de parangón a la normatividad anteriormente expresada. Desde esta perspectiva, la

6. Queremos decir con ello, que cuando ubiquemos, por ejemplo, la Sociología de la Educación lo haremos en el mismo lugar en que tendría que estar ubicada la Sociología. En todo caso diferenciaremos ambos posicionamientos a través de la diferente grafía, por lo que utilizaremos la negrita para dar a entender la ubicación de la ciencia humana de la que se trate. Lo mismo haremos con el caso de la Biología que, obviamente, si bien se refiere al hombre, no forma parte, en sentido estricto, de lo que se entiende por Ciencias Humanas. No obstante, su inclusión obedece, además de a su tradición, a la funcionalidad que juega, pareja a la de las Ciencias Humanas y nunca porque la consideremos una más de entre ellas. 
filosofía, la política..., etc., y sobre todo sus manifestaciones referidas a la cuestión teleológica, ética y axiológica de la educación, se refrendarían como materias fundamentales propias de tal dimensión.

Atendiendo a todo ello, el diagrama que reflejaría la ubicación de las Ciencias de la Educación (en relación a las propias ciencias humanas, como un ejemplo casi perfecto de concomitancia), según la división piagetiana de las mismas, sería el siguiente:

CUADRo 3. Exoestructura de las Ciencias de la Educación.

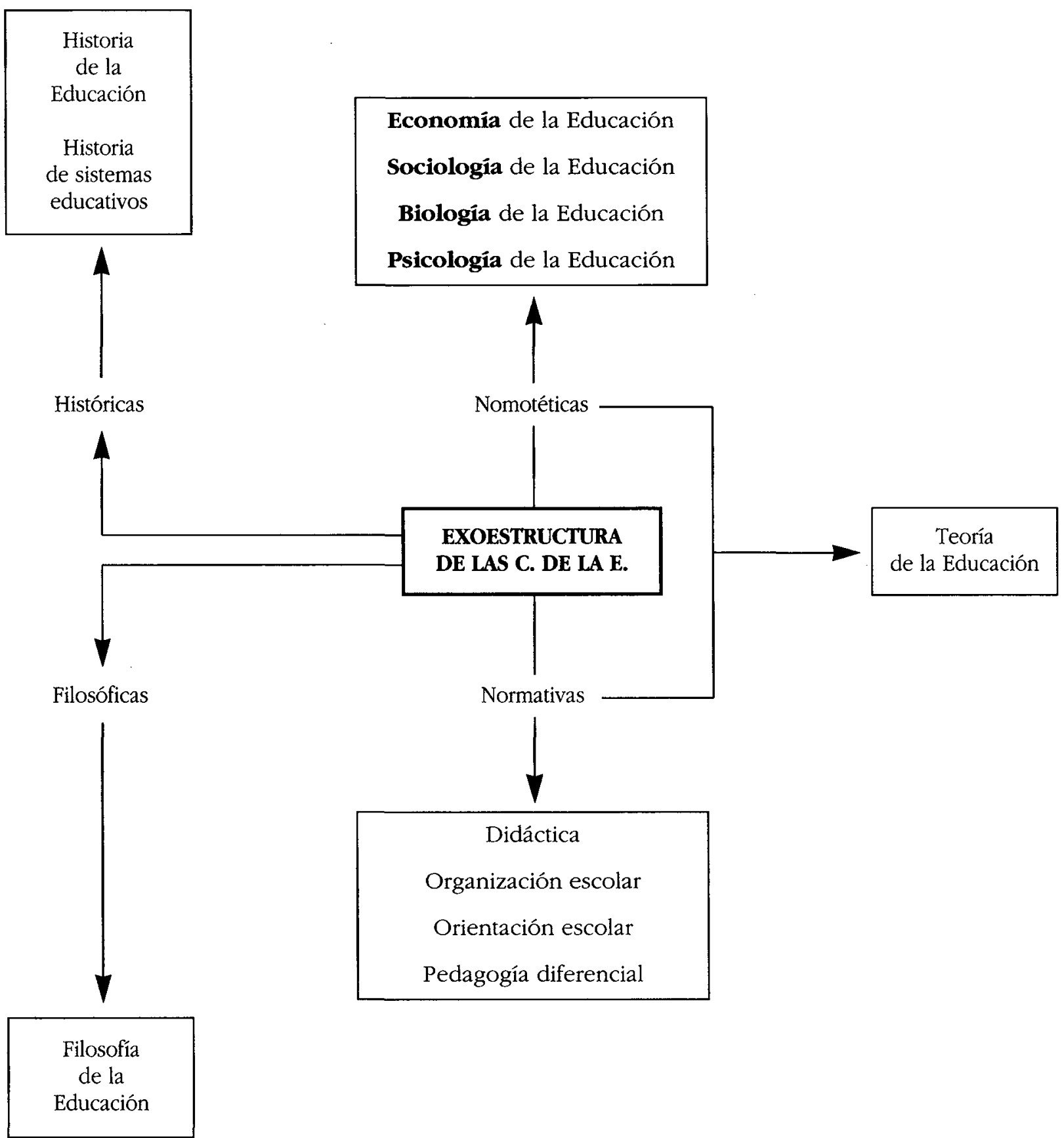


ANTONIO J. COLOM CANELLAS Y M $M^{a}$ DEL PINO RODRÍGUEZ CRUZ

\section{REFLEXIÓN FINAL}

Esta concomitancia entre las Ciencias de la Educación y las ciencias humanas podría resumirse en dos enfoques: uno de subsidariedad y el otro de complementariedad.

Subsiedaridad, porque dada la complejidad del proceso educativo, la aportación de las ciencias humanas, en especial la Psicología y la Sociología, constituye la plataforma ideal para reconsiderar científicamente la cuestión educativa, y en consecuencia, para redefinir cualquier planteamiento educativo. Al mismo tiempo, cabe también hablar de complementariedad, porque al estar el fenómeno educativo presente en cualquier manifestación de las diversas ciencias humanas, aquellos planteamientos efectuados en función de la subsiedaridad, se integran en el ámbito de la ciencia humana que les son propios, orientando nuevas perspectivas, reformulando viejas conclusiones, y en definitiva, enriqueciendo y redifiniendo cuestiones y aspectos intrínsecos a las mismas.

Ubicadas pues adecuadamente las Ciencias de la Educación entre las ciencias humanas, interesa concluir que si bien las Ciencias de la Educación constituyen un campo abierto a aportaciones de todas las ciencias que se interesen por la cuestión educativa - posición de subsiedaridad-, en la medida que la actividad educativa intencional crea nuevas opciones, en la medida en que el dinamismo inherente a esa actividad educativa pueda romper caducos esquemas y crear nuevos planteamientos y paradigmas, en esta medida, insistimos, las ciencias humanas están también condicionadas y complementadas por las Ciencias de la Educación.

O dicho de otro modo, en las Ciencias de la Educación concurren todas las ciencias humanas, y por el contrario, en todas ellas se puede descubrir una dimensión educativa. Es en esta articulación en donde deben descansar cualquier posicionamiento respecto a la consistencia y ubicación de las Ciencias de la Educación. Y es también, en este punto, en donde cualquier crítica a la consistencia epistemológica de las Ciencias de la Educación queda diluida porque un posicionamiento de tal índole supondría al mismo tiempo una crítica a las ciencias humanas y en consecuencia, a la capacidad comprensiva que el hombre tiene respecto de los fenómenos que concurren en su dimensión fenomenológica. Hablar de las Ciencias de la Educación no es más que especializar, en función del objeto educativo, una parcela de las ciencias humanas, y viceversa, hablar de las Ciencias Humanas es referirse implícitamante al contexto conceptual que cobija a la educación y su estudio, en toda su dimensionalidad y pluralidad de desarrollos.

Por su parte, el posicionamiento de la Teoría de la Educación en el amplio espectro de las ciencias humanas es claro y no ofrece ahora duda alguna. Su ubicación entre ellas, es idéntico a la situación en la que se encontraba dentro del marco de las Ciencias de la Educación, porque en definitiva, entre ellas, entre ciencias humanas y ciencias de la educación, se da, como hemos visto, una relación absolutamente concomitante ya que se trata de un mismo contexto conceptual al conformarse las Ciencias de la Educación como una parcela especializada de las ciencias humanas. La Teoría de la Educación, por tanto, bien en el seno de las Ciencias de la Educación, o bien en la relación de estas con las ciencias huma- 
nas, se nos aparece siempre directamente incidida por la perspectiva nomotética o científica y por los vectores normativos o de practicidad 7 . Ello hace que aboguemos una vez más por el carácter teórico-tecnológico de la Teoría de la Educación, o si se quiere, por entender el sentido de su sustantividad teórica como teoría para la práctica. Con ello la Teoría de la Educación muestra a los demás desarrollos propios de las ciencias humanas un nuevo modelo conceptual mixto, a caballo entre el saber y el hacer, que en un futuro no muy lejano será tenido en cuenta por otras ciencias humano-sociales, visto el desarrollo de la sociedad y el papel que la técnica y la tecnología están jugando en ella.

\section{BiBliogRAFía}

Colom, A. J. (1982): Teoría y metateoría de la educación, Trillas, México.

Colom, A. J. (1992): El saber de la teoría de la educación, Teoría de la Educación, vol. 4, pp. 11 a 19.

DuRKHEIM, E. (1975): Educación y Sociología, Península, Barcelona.

PÉRez Gómez, A. (1978): Las fronteras de la educación, Edit. Zero, ZYX, Madrid.

PIAGeT, J. (1969): Psicología y Pedagogía, Ariel, Barcelona.

7. Indirectamente la teoría recibe aportes de las disciplinas filosóficas, históricas, etc. que de alguna forma condicionan los paradigmas teórico-prácticos o científico-normativos que definen su idiosincracia. 